\title{
Perovskite LEDs
}

\section{Richard H. Friend, ${ }^{1 *}$ Dawei $\mathrm{Di}^{2,{ }^{2 *}}$ Samuele Lilliu, ${ }^{3}$ Baodan Zhao ${ }^{1}$}

Sir Richard H. Friend is Cavendish Professor of Physics at the University of Cambridge. In the 1990s, he reported for the first time efficient operation of polymer based FET and LED, which contributed to the commercialisation of OLED displays employed in current TV and smartphone devices. He is co-founder of several companies and start-ups including Cambridge Display Technology, Plastic Logic, and Heliochrome limited. In this interview, he and Dr. Dawei Di, who recently joined Zhejiang University in China as a tenure-track professor, discuss recent developments and future prospects of perovskite LED research and development. The interview is available at https://youtu.be/Fjcm4V36U2A.

\section{Background}

Samuele Lilliu (SL): Richard, you have been one of the pioneers in the field of organic electronics. Can you talk about the path that has led from your initial works on polymer light emitting diodes to the current OLED technology used in consumer electronics?

Richard H. Friend (RHF): In the mid-1980s I, along with quite a few others, was interested in the conducting properties of some of the so-called organic metals and I realised that the improvements in the materials that were being synthesized meant that we could start thinking about their use in semiconductor devices. ${ }^{1-7} \mathrm{I}$ did set out, very deliberately, to create a program to make semiconductor devices and our first big success was demonstrating transistors that work extremely well and actually worked in an interesting way. The nature of the field-induced charges is quite different to what one usually finds in an inorganic semiconductor. We had a big success with a paper we published in Nature in $1988 .^{8}$ That really projected our work and the field in general into the public view.

We were looking around for other things to do and explored other materials and we, more or less by well-prepared chance, discovered that simple diode structures, i.e. a stack structure with an electrode, a layer of semiconductor, and an electrode on top, produced quite good devices, but rather unexpectedly, showed light emission, LED operation. That was the work of quite a few of us. Among the co-authors on the paper we published in Nature in $1990,{ }^{9}$ I pick out Jeremy Burroughes, who in the end stayed all the way through Cambridge Display Technology, Donald Bradley, who set an extremely successful career away from Cambridge, Andrew Holmes, who was then my chemistry colleague in Cambridge and has had a very successful career later both at Cambridge and now back in Australia, and certainly Paul Burns, who was a postdoc at that time in Cambridge and is now professor back in Australia. So, a lot of us were around and the right things were done and we discovered we could get light out of some polymers. There were antecedent demonstrations of electroluminescence, not from polymers but from so-called single molecules, which in relation to our polymer device produced by solution processing looked very important. ${ }^{10}$

We filed a patent, ${ }^{11}$ which turned out to be a whole embracing patent that was very valuable. Around that we raised money in interesting ways (some of the initial investors were Cambridge Research and Innovation ltd, Cambridge University, and 'business angels' such as members of the rock group Genesis). ${ }^{12}$ Back in the early 1990s there was not a lot of venture capital around. We ended up with Cambridge Display Technology ${ }^{13}$ as a company with a

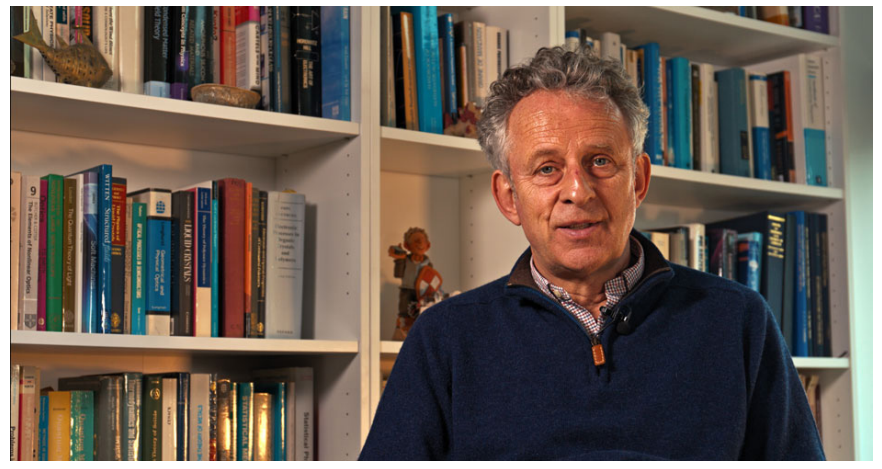

Figure 1 | Prof. Sir Richard H. Friend, Cavendish Professor of Physics.

strong university involvement, a university shareholding, which we evolved to be able to prove the manufacturability of the science discovery we had made. There are a number of steps along the way that were proven very important. We had a collaboration in the mid-1990s with Seiko Epson, who of course make up some inkjet printers and through that we developed ways to directly pattern red, green, and blue subpixels by direct printing. ${ }^{12}$ That has, decades later, proved to be a very practical way of producing large area OLED screens. They are now available commercially.

Along the way the company acquired a number of different shareholders and was eventually acquired by the Sumitomo Chemical company, ${ }^{14}$ who have sustained the R\&D activity in Cambridge, but of course has done a lot of commercialisation in the Japanese operation. That deep pocket and long term ambition has been critical to success of that commercialisation.

\section{Current Progress of Perovskite LEDs}

SL: Perovskite materials have emerged as the new technology for solar cells and the number of publications has skyrocketed since 2012. ${ }^{15-22}$ There seems to be less groups involved in perovskite LEDs. Can you talk about the perovskite LED technology and how this compares to established OLED technology?

RHF: The first point to make is that LEDs are slightly more difficult to make than solar cells and certainly more difficult to measure and they naturally attract a slightly more specialised researcher community. The number of papers published on perovskite LEDs is rather large. ${ }^{23-26}$ I think our paper that we published in $2014^{27}$ has had well over a thousand citations, which indicates that it is actually

${ }^{1 *}$ Cavendish Laboratory, University of Cambridge, Cambridge, UK, Contact R.H. Friend, ${ }^{2}$ State Key Laboratory of Modern Optical Instrumentation, College of Optical Science and Engineering, Zhejiang University, Hangzhou, China, Contact Dawei Di, ${ }^{3}$ Department of Physics and Astronomy, University of Sheffield, Sheffield, UK 
quite a large community working on it, not as large as the solar cells', but by ordinary measures it is a very busy community.

Organic LEDs which have been under serious commercial development since the early 1990s, now perform quite well. They are used for top-end televisions and top-end smart phones. ${ }^{28-32}$ There are actually two slightly different technologies used for these two applications. It is certainly salutary that it has taken essentially three decades to engineer that technology to provide what is truly excellent quality, and it is now firmly established as the best current display technology. OLEDs have some limitations. Ultimately the lifetime is limited. ${ }^{33-37}$ There is an issue always with one colour, the blue, which has always been the hardest colour to optimise for OLEDs, but it is adequate for televisions. ${ }^{38,39}$ There is also a question about their efficiency in converting electrical power to light energy. I think they now perform like crystal displays, ${ }^{40}$ they should do, and their power efficiency is almost certainly one of the reasons why they are widely adopted in smart phones. ${ }^{41}$ There are some limitations to the amount of light you can bring out in the forward direction from a flat panel display. ${ }^{32}$ We have ideas, which we have not really made work yet, but we think are viable to push the performance of perovskite LEDs. There are some particularities about how the semiconducting optoelectronic properties operate that may allow us to get better light extraction ${ }^{42}$ in the forward direction than OLED technology and that is one of the reasons why we think it is worth exploring. ${ }^{43}$

SL: In November 2018 your group published a paper in Nature Photonics ${ }^{43}$ on the most efficient perovskite LEDs reported to date. Can you talk about how did you come up with the design of the perovskite-polymer bulk heterostructure $(\mathrm{PPBH})$ ?

RHF: The challenge with any thin film LED technology is that we need to do two things at the same time. The first is that we need to inject electrons from one side and holes from the other and cause them to recombine in the active semiconductor. The second is that we then want that excitation to emit a photon, to produce light rather than cause heat to be generated through accidental non-radiative recombination. In general interfaces between the luminescent material and the injection layers, are difficult to engineer to produce really good luminescent behaviour. It is quite often the case that there is quenching. ${ }^{44-47}$ After our original result that we published in $2014,{ }^{27}$ we planned to engineer materials or composite materials, where the light emission takes places relatively deep within the layer of the perovskite, so that the electrodes, which needed to inject charges are not able to act as quenching sites. This was achieved in our recent paper in Nature Photonics ${ }^{43}$ by using a mixture of perovskite materials with different colours, with different bandgaps, and to contain that mixture within a polymer matrix. There is an element of empirical optimisation, but the design rule was very clear. We knew that if we did not have a very high photoluminescence yield, we would not have an optimum electroluminescence yield.

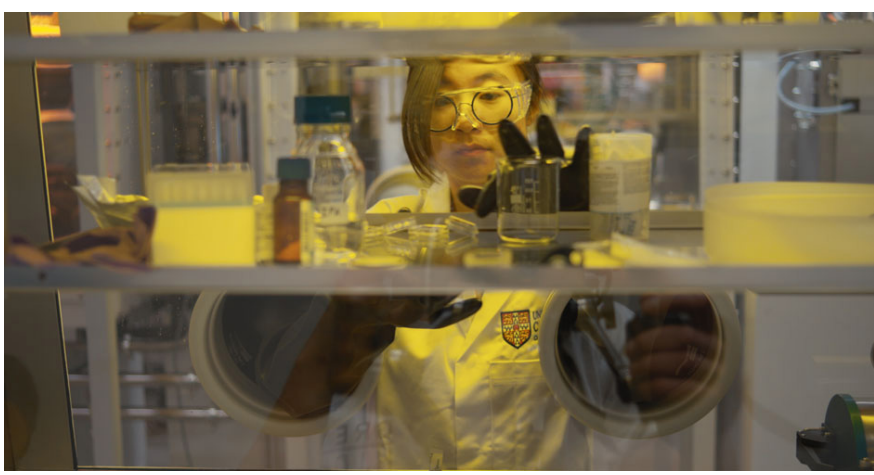

Figure 2 | Baodan Zhao, PhD student and first author of ref. [43], at the Maxwell Centre, Cavendish Laboratory.

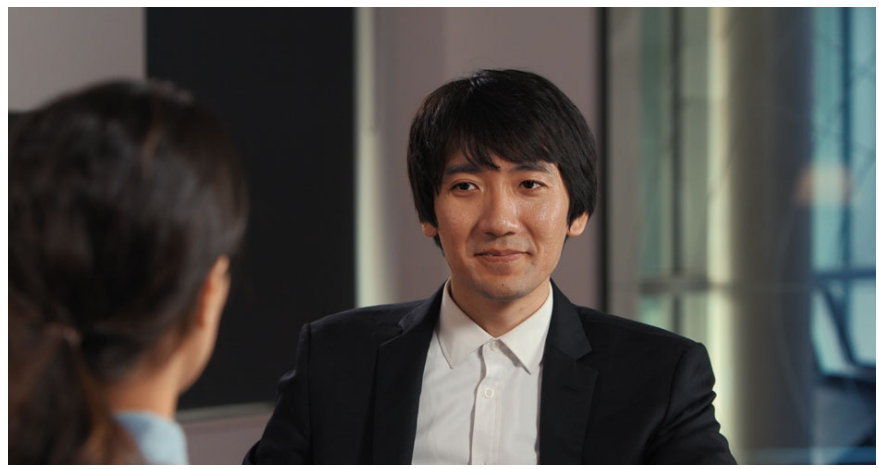

Figure 3 | Dr Dawei Di, professor at the State Key Laboratory of Modern Optical Instrumentation, College of Optical Science and Engineering, Zhejiang University (Hangzhou, China) and visiting researcher at the Cavendish Laboratory.

Mejd Alsari (MA): Dawei, in your recent Nature photonics paper you have demonstrated perovskite LEDs with EQE exceeding 20\%, would you like to share your thoughts about this report?

Dawei Di (DD): In our recent publication with Prof. Richard Friend, Baodan Zhao and colleagues, ${ }^{43}$ we employ a bulk heterostructure or blend of quasi-2D/3D perovskites ${ }^{48,49}$ and an insulating polymer to confine charge carriers and eliminate non-radiative recombination losses at the same time. We have developed some of the highest-efficiency perovskite LEDs, with external quantum efficiencies exceeding 20\%. This means that the efficiencies of perovskite LEDs are now on par with some of the best OLEDs and quantum-dot LEDs..$^{50-52}$

Through transient optical measurements, we found that the charge-transport interfaces have no observable influence on the photoluminescence (PL) kinetics of the emissive layer. This highly desirable condition has been achieved for the first time for perovskite devices including LEDs and solar cells. It means that the interfacial recombination losses are effectively supressed. The external PL quantum yield of the emissive layer are close to $100 \%$, indicating the elimination of non-radiative losses.

Ultrafast optical experiments show that the photo-excited states initially formed in the higher-bandgap quasi-2D regions quickly migrate to lower-bandgap $3 \mathrm{D}$ regions within $1 \mathrm{ps}$, followed by radiative bimolecular recombination in the $3 \mathrm{D}$ perovskite region. The carrier lifetime in the 3D perovskite region is enhanced due to the presence of the polymer. Together, the two processes ensure that non-radiative losses in the emissive layer are effectively eliminated.

Our calculations based on conventional optical models for thinfilm OLEDs suggest that the internal electroluminescence quantum efficiencies of our best LEDs are close to $100 \%$. We believe further improvements of external electroluminescence efficiencies could be possible by using light extraction strategies including photon scattering and recycling.

\section{Future Developments of Perovskite LEDs}

MA: Can you comment on future developments?

DD: One of the advantages of perovskite light emitters is the high colour purity. On the other hand this is also a drawback, because if we want to realise white emission with high colour rendering index (CRI) this is going to be quite difficult. There is a recent publication in Nature by a Chinese group headed by Prof. Jiang Tang, where they demonstrate some interesting white emitting perovskite composition..$^{53}$ So I think perovskites are also capable of emitting white light efficiently. If we make use of the white emitting perovskites and make them into efficient LEDs they also have very good potential for lighting purposes with high CRI. 
SL: Richard, How do you think these devices can be further improved?

RHF: The opportunity going forward is obviously to improve lifetime, the manufacturing window, and to control colour. We reported near-infrared devices in that paper. ${ }^{43}$ We have competing groups around the world that have been producing emission with other colours. ${ }^{54-57}$ We know now how to achieve comparable performance with very good green devices. We need to examine protocols for producing large areas or patterned structures. We know we will have some challenges in getting an excellent blue device. That is always the difficult colour. There is a lot to do, but the important point about our paper, and there were similar papers from groups elsewhere around the world at about the same time that were published, ${ }^{54-57}$ is that it demonstrates that the architecture and the materials look very promising. The performance of $20 \%$ external quantum efficiency in the forward direction is a sort of benchmark that establishes these as serious contenders for application. What would be really exciting is to push the performance beyond the performance that is achieved in today's technologies for thin film displays.

SL: Recent works in solar cells suggest that the degradation of perovskites is not an issue for certain mixed cation materials. What are the challenges that perovskite LEDs face to enter the market?

RHF: With almost all new semiconductor technologies it has taken time to develop reliability. That has been true for III-V semiconductor lasers, organic LEDs, and it will be true for any new solar technology. With the perovskite materials there has been a lot of industrial effort to improve the stability of solar cells and the results are now encouraging. With the light-emitting diodes, the work at the moment that I am aware of is purely academic and the efforts that have been made have been more towards demonstrating the potential for efficient operation rather than engineer long lived devices. There are some interesting opportunities to operate the devices in a regime where we should be able to enjoy the same stability that is now demonstrated for solar cells. It is actually operation in an interesting and slightly different mode to that usually employed in perovskite devices. The key to running perovskite devices is to arrange that the only currents flowing in the perovskite semiconductor are diffusion currents rather than drift currents. ${ }^{58,59}$ That means that we need to avoid trying to maintain an electric field across the perovskite semiconductor. I think that is satisfactory for the operation of these devices. LEDs?

SL: How can you achieve white light emission with perovskites

RHF: The requirement for a display is to try to produce red, green, and blue pixels that are the vertices of the colour triangle. We want very pure narrow lines with colours that in combination would produce any colour as perceived by the human eye. In contrast if we want to produce white light for lighting, we actually need to mimic the black body spectrum that we get from sunlight or tungsten light bulbs and we need to fill in all wavelengths. Current LED lighting based on $\mathrm{GaN}$, which is blue, use some relatively cheap phosphors that sort of fill in the other colours, but not perfectly. ${ }^{60}$ There is a really interesting opportunity to build in a composite of different phosphors that each of which take some of the blue light from the GaN LED and absorbs it and re-emits is at a downshifted colour, such as green, orange, yellow, or red. ${ }^{61-63}$ The likely optimization would involve a sort of recipe of several materials in combination producing a very good match of what we are familiar with the sun.

SL. Are you aware of any company working on perovskite LEDs?

RHF. In Cambridge we have filed patents around perovskite LEDs and around the luminescent properties. ${ }^{64-66}$ We have been working together with the Oxford group, with Henry Snaith, and we have secured some venture capital funding and we have created a company, which is Heliochrome Limited, ${ }^{67}$ which we will use as a body vehicle to continue to build up intellectual property and to explore a number of possible application areas for luminescent perovskites and LEDs made with them.

\section{References}

1 R. H. Friend, S. Rehmatullah, A. D. Yoffe \& D. Jérome. Electrical conductivity in polymeric sulphur nitride at high pressures. Journal of Physics C: Solid State Physics 10, 1001-1012, (1977).

2 R. H. Friend, D. Jerome, J. M. Fabre, L. Giral \& K. Bechgaard. Stabilisation of the metallic state at low temperatures in HMTTF-TCNQ under pressure. Journal of Physics C: Solid State Physics 11, 263-275, (1978).

3 R. H. Friend, M. Miljak \& D. Jérome. Pressure Dependence of the Phase Transitions in Tetrathiafulvalene-Tetracyanoquinodimethane (TTF-TCNQ): Evidence for a Longitudinal Lockin at 20 kbar. Physical Review Letters 40, 10481051, (1978).

4 W. J. Feast, I. S. Millichamp, R. H. Friend, M. E. Horton, D. Phillips, S. D. D. V. Rughooputh \& G. Rumbles. Optical absorption and luminescence in poly(4,4'-diphenylenediphenylvinylene). Synthetic Metals 10, 181-191, (1985).

5 D. D. C. Bradley, R. H. Friend, H. Lindenberger \& S. Roth. Infra-red characterization of oriented poly(phenylene vinylene). Polymer 27, 1709-1713, (1986).

6 D. D. C. Bradley, G. P. Evans \& R. H. Friend. Characterisation of poly (phenylenevinylene) by infrared and optical absorption. Synthetic Metals 17, 651656, (1987).

7 R. H. Friend, D. D. C. Bradley \& P. D. Townsend. Photo-excitation in conjugated polymers. Journal of Physics D: Applied Physics 20, 1367-1384, (1987).

8 J. H. Burroughes, C. A. Jones \& R. H. Friend. New semiconductor device physics in polymer diodes and transistors. Nature 335, 137, (1988).

9 J. H. Burroughes, D. D. C. Bradley, A. R. Brown, R. N. Marks, K. Mackay, R. H. Friend, P. L. Burns \& A. B. Holmes. Light-emitting diodes based on conjugated polymers. Nature 347, 539, (1990).

10 C. W. Tang \& S. A. VanSlyke. Organic electroluminescent diodes. Applied Physics Letters 51, 913-915, (1987).

11 R. H. Friend, J. H. Burroughes \& D. D. C. Bradley. Electroluminescent devices, https://patents.google.com/patent/US5247190A/en, (1989).

12 T. I. M. Minshall, S. Seldon \& D. Probert. Commercializing a disruptive technology based upon university IP through open innovation: a case study of Cambridge Display Technology. International Journal of Innovation and Technology Management 04, 225-239, (2007).

13 Cambridge Display Technology, https://www.cdtltd.co.uk/, (2019).

14 Sumitomo Chemical to Acquire Cambridge Display Technology Inc., https:// www.sumitomo-chem.co.jp/english/news/files/docs/20070731 3.pdf, (2007).

15 M. A. Green, A. Ho-Baillie \& H. J. Snaith. The emergence of perovskite solar cells. Nature Photonics 8, 506, (2014).

16 H. J. Snaith. Present status and future prospects of perovskite photovoltaics. Nature Materials 17, 372-376, (2018).

17 M. Liu, M. B. Johnston \& H. J. Snaith. Efficient planar heterojunction perovskite solar cells by vapour deposition. Nature 501, 395, (2013).

18 H. Zhou, Q. Chen, G. Li, S. Luo, T.-b. Song, H.-S. Duan, Z. Hong, J. You, Y. Liu \& Y. Yang. Interface engineering of highly efficient perovskite solar cells. Science 345, 542-546, (2014).

19 H. J. Snaith \& S. Lilliu. The Path to Perovskite on Silicon PV. Scientific Video Protocols 1, 1, (2018).

20 N. J. Jeon, J. H. Noh, Y. C. Kim, W. S. Yang, S. Ryu \& S. I. Seok. Solvent engineering for high-performance inorganic-organic hybrid perovskite solar cells. Nature Materials 13, 897, (2014).

21 M. M. Lee, J. Teuscher, T. Miyasaka, T. N. Murakami \& H. J. Snaith. Efficient Hybrid Solar Cells Based on Meso-Superstructured Organometal Halide Perovskites. Science 338, 643-647, (2012).

22 J. Burschka, N. Pellet, S.-J. Moon, R. Humphry-Baker, P. Gao, M. K. Nazeeruddin \& M. Grätzel. Sequential deposition as a route to high-performance perovskite-sensitized solar cells. Nature 499, 316, (2013).

23 H. Cho, S.-H. Jeong, M.-H. Park, Y.-H. Kim, C. Wolf, C.-L. Lee, J. H. Heo, A. Sadhanala, N. Myoung, S. Yoo, S. H. Im, R. H. Friend \& T.-W. Lee. Overcoming the electroluminescence efficiency limitations of perovskite light-emitting diodes. Science 350, 1222-1225, (2015).

24 Y.-H. Kim, H. Cho, J. H. Heo, T.-S. Kim, N. Myoung, C.-L. Lee, S. H. Im \& 
T.-W. Lee. Multicolored Organic/Inorganic Hybrid Perovskite Light-Emitting Diodes. Advanced Materials 27, 1248-1254, (2015).

25 J. Song, J. Li, X. Li, L. Xu, Y. Dong \& H. Zeng. Quantum Dot Light-Emitting Diodes Based on Inorganic Perovskite Cesium Lead Halides (CsPbX3). Advanced Materials 27, 7162-7167, (2015).

26 J. Wang, N. Wang, Y. Jin, J. Si, Z.-K. Tan, H. Du, L. Cheng, X. Dai, S. Bai, H. He, Z. Ye, M. L. Lai, R. H. Friend \& W. Huang. Interfacial Control Toward Efficient and Low-Voltage Perovskite Light-Emitting Diodes. Advanced Materials 27, 2311-2316, (2015).

27 Z.-K. Tan, R. S. Moghaddam, M. L. Lai, P. Docampo, R. Higler, F. Deschler, M. Price, A. Sadhanala, L. M. Pazos, D. Credgington, F. Hanusch, T. Bein, H. J. Snaith \& R. H. Friend. Bright light-emitting diodes based on organometal halide perovskite. Nature Nanotechnology 9, 687, (2014).

D. Volz, M. Wallesch, C. Fléchon, M. Danz, A. Verma, J. M. Navarro, D. M. Zink, S. Bräse \& T. Baumann. From iridium and platinum to copper and carbon: new avenues for more sustainability in organic light-emitting diodes. Green Chemistry 17, 1988-2011, (2015)

29 C. Adachi, M. A. Baldo, S. R. Forrest \& M. E. Thompson. High-efficiency organic electrophosphorescent devices with tris(2-phenylpyridine)iridium doped into electron-transporting materials. Applied Physics Letters 77, 904 906, (2000).

30 S. R. Forrest, P. E. Burrows, Z. Shen, G. Gu, V. Bulovic \& M. E. Thompson. The stacked OLED (SOLED): a new type of organic device for achieving high-resolution full-color displays. Synthetic Metals 91, 9-13, (1997).

31 Q. Huang, R. Meerheim, K. Fehse, G. Schwartz, S. Reineke, K. Walzer \& K. Leo. 36-1: Invited Paper: 2nd Generation Organics: High Power Efficiency, Ultra Long Life, and Low-Cost OLED Devices. SID Symposium Digest of Technical Papers 38, 1282-1285, (2007)

32 J.-H. Jou, S. Kumar, A. Agrawal, T.-H. Li \& S. Sahoo. Approaches for fabricating high efficiency organic light emitting diodes. Journal of Materials Chemistry C 3, 2974-3002, (2015).

33 S.-G. Ihn, N. Lee, S. O. Jeon, M. Sim, H. Kang, Y. Jung, D. H. Huh, Y. M. Son, S Y. Lee, M. Numata, H. Miyazaki, R. Gómez-Bombarelli, J. Aguilera-Iparraguirre, T. Hirzel, A. Aspuru-Guzik, S. Kim \& S. Lee. An Alternative Host Material for Long-Lifespan Blue Organic Light-Emitting Diodes Using Thermally Activated Delayed Fluorescence. Advanced Science 4, 1600502, (2017).

34 A. Laaperi. OLED lifetime issues from a mobile-phone-industry point of view. Journal of the Society for Information Display 16, 1125-1130, (2008).

35 C. Jeong, C. Coburn, M. Idris, Y. Li, P. I. Djurovich, M. E. Thompson \& S. R. Forrest. Understanding molecular fragmentation in blue phosphorescent organic light-emitting devices. Organic Electronics 64, 15-21, (2019).

36 J.-H. Lee, H. Shin, J.-M. Kim, K.-H. Kim \& J.-J. Kim. Exciplex-Forming CoHost-Based Red Phosphorescent Organic Light-Emitting Diodes with Long Operational Stability and High Efficiency. ACS Applied Materials \& Interfaces 9, 3277-3281, (2017)

37 Z.-Q. Zhu, K. Klimes, S. Holloway \& J. Li. Efficient Cyclometalated Platinum(II) Complex with Superior Operational Stability. Advanced Materials 29, 1605002, (2017).

38 Q. Zhang, B. Li, S. Huang, H. Nomura, H. Tanaka \& C. Adachi. Efficient blue organic light-emitting diodes employing thermally activated delayed fluorescence. Nature Photonics 8, 326, (2014).

39 S. Wu, M. Aonuma, Q. Zhang, S. Huang, T. Nakagawa, K. Kuwabara \& C. Adachi. High-efficiency deep-blue organic light-emitting diodes based on a thermally activated delayed fluorescence emitter. Journal of Materials Chemistry C 2, 421-424, (2014).

40 OLED Versus LCD: Who Wins?, http://lcd.creol.ucf.edu/Publications/2015/19-21-OptInnov-Feb15.pdf, (2015).

41 Life-Cycle Assessment of Energy and Environmental Impacts of LED Lighting Products https://www.pnnl.gov/main/publications/external/technical_reports/PNNL-21443.pdf, (2012)

42 K. Saxena, V. K. Jain \& D. S. Mehta. A review on the light extraction techniques in organic electroluminescent devices. Optical Materials 32, 221-233, (2009)

43 B. Zhao, S. Bai, V. Kim, R. Lamboll, R. Shivanna, F. Auras, J. M. Richter, L. Yang, L. Dai, M. Alsari, X.-J. She, L. Liang, J. Zhang, S. Lilliu, P. Gao, H. J. Snaith, J. Wang, N. C. Greenham, R. H. Friend \& D. Di. High-efficiency per- ovskite-polymer bulk heterostructure light-emitting diodes. Nature Photonics 12, 783-789, (2018).

44 J. M. Ball \& A. Petrozza. Defects in perovskite-halides and their effects in solar cells. Nature Energy 1, 16149, (2016)

45 A. Walsh \& A. Zunger. Instilling defect tolerance in new compounds. Nature Materials 16, 964, (2017).

46 D. Meggiolaro, S. G. Motti, E. Mosconi, A. J. Barker, J. Ball, C. Andrea Riccardo Perini, F. Deschler, A. Petrozza \& F. De Angelis. Iodine chemistry determines the defect tolerance of lead-halide perovskites. Energy \& Environmental Science 11, 702-713, (2018).

47 A. Zakutayev, C. M. Caskey, A. N. Fioretti, D. S. Ginley, J. Vidal, V. Stevanovic, E. Tea \& S. Lany. Defect Tolerant Semiconductors for Solar Energy Conversion. The Journal of Physical Chemistry Letters 5, 1117-1125, (2014).

48 M. Yuan, L. N. Quan, R. Comin, G. Walters, R. Sabatini, O. Voznyy, S. Hoogland, Y. Zhao, E. M. Beauregard, P. Kanjanaboos, Z. Lu, D. H. Kim \& E. H. Sargent. Perovskite energy funnels for efficient light-emitting diodes. Nature Nanotechnology 11, 872, (2016).

49 N. Wang, L. Cheng, R. Ge, S. Zhang, Y. Miao, W. Zou, C. Yi, Y. Sun, Y. Cao, R. Yang, Y. Wei, Q. Guo, Y. Ke, M. Yu, Y. Jin, Y. Liu, Q. Ding, D. Di, L. Yang, G. Xing, H. Tian, C. Jin, F. Gao, R. H. Friend, J. Wang \& W. Huang. Perovskite light-emitting diodes based on solution-processed self-organized multiple quantum wells. Nature Photonics 10, 699, (2016).

50 S. Reineke, F. Lindner, G. Schwartz, N. Seidler, K. Walzer, B. Lüssem \& K. Leo. White organic light-emitting diodes with fluorescent tube efficiency. Nature 459, 234, (2009).

51 H. Uoyama, K. Goushi, K. Shizu, H. Nomura \& C. Adachi. Highly efficient organic light-emitting diodes from delayed fluorescence. Nature 492, 234, (2012).

52 D. Di, A. S. Romanov, L. Yang, J. M. Richter, J. P. H. Rivett, S. Jones, T. H. Thomas, M. Abdi Jalebi, R. H. Friend, M. Linnolahti, M. Bochmann \& D. Credgington. High-performance light-emitting diodes based on carbene-metal-amides. Science 356, 159-163, (2017).

53 J. Luo, X. Wang, S. Li, J. Liu, Y. Guo, G. Niu, L. Yao, Y. Fu, L. Gao, Q. Dong, C. Zhao, M. Leng, F. Ma, W. Liang, L. Wang, S. Jin, J. Han, L. Zhang, J. Etheridge, J. Wang, Y. Yan, E. H. Sargent \& J. Tang. Efficient and stable emission of warmwhite light from lead-free halide double perovskites. Nature 563, 541-545, (2018).

54 K. Lin, J. Xing, L. N. Quan, F. P. G. de Arquer, X. Gong, J. Lu, L. Xie, W. Zhao, D. Zhang, C. Yan, W. Li, X. Liu, Y. Lu, J. Kirman, E. H. Sargent, Q. Xiong \& Z. Wei. Perovskite light-emitting diodes with external quantum efficiency exceeding 20 per cent. Nature 562, 245-248, (2018)

55 Y. Cao, N. Wang, H. Tian, J. Guo, Y. Wei, H. Chen, Y. Miao, W. Zou, K. Pan, Y. He, H. Cao, Y. Ke, M. Xu, Y. Wang, M. Yang, K. Du, Z. Fu, D. Kong, D. Dai, Y. Jin, G. Li, H. Li, Q. Peng, J. Wang \& W. Huang. Perovskite light-emitting diodes based on spontaneously formed submicrometre-scale structures. Nature 562, 249-253, (2018).

56 J. Xing, Y. Zhao, M. Askerka, L. N. Quan, X. Gong, W. Zhao, J. Zhao, H. Tan, G. Long, L. Gao, Z. Yang, O. Voznyy, J. Tang, Z.-H. Lu, Q. Xiong \& E. H. Sargent. Color-stable highly luminescent sky-blue perovskite light-emitting diodes. Nature Communications 9, 3541, (2018).

57 T. Chiba, Y. Hayashi, H. Ebe, K. Hoshi, J. Sato, S. Sato, Y.-J. Pu, S. Ohisa \& J. Kido. Anion-exchange red perovskite quantum dots with ammonium iodine salts for highly efficient light-emitting devices. Nature Photonics 12, 681-687, (2018).

58 H. Kim, L. Zhao, J. S. Price, A. J. Grede, K. Roh, A. N. Brigeman, M. Lopez, B. P. Rand \& N. C. Giebink. Hybrid perovskite light emitting diodes under intense electrical excitation. Nature Communications 9, 4893, (2018).

59 G. Richardson, S. E. J. O’Kane, R. G. Niemann, T. A. Peltola, J. M. Foster, P. J. Cameron \& A. B. Walker. Can slow-moving ions explain hysteresis in the current-voltage curves of perovskite solar cells? Energy \& Environmental Science 9, 1476-1485, (2016).

60 R. D. Dupuis \& M. R. Krames. History, Development, and Applications of High-Brightness Visible Light-Emitting Diodes. Journal of Lightwave Technology 26, 1154-1171, (2008).

61 E. F. Schubert \& J. K. Kim. Solid-State Light Sources Getting Smart. Science 308, 1274-1278, (2005) 
62 N. C. George, K. A. Denault \& R. Seshadri. Phosphors for Solid-State White Lighting. Annual Review of Materials Research 43, 481-501, (2013).

63 X. Huang. Red phosphor converts white LEDs. Nature Photonics 8, 748, (2014).

64 R. H. Friend, G. Li, D. DI, R. S. Moghaddam \& Z. K. Tan. Luminescent device, https://patents.google.com/patent/WO2017001542A1/en?oq=perovskite+light+emitting+diode+friend, (2015).

65 R. H. Friend, Z. K. Tan, A. Sadhanala, M. L. Lai, P. Docampo, F. Deschler, M. Price, F. Hanusch, H. Snaith \& R. S. Moghaddam. Electroluminescent device, $\quad$ https://patents.google.com/patent/CA2947381A1/en?oq=perovskite+light+emitting+diode+friend, (2014).

66 R. H. Friend, Z. K. Tan, L. Guangru, D. Di \& N. C. Greenham Electroluminescent device, https://patents.google.com/patent/US20170324057A1/en, (2015).

67 Heliochrome limited, https://beta.companieshouse.gov.uk/company/10498672, (2016).

Conflicts of Interest

R.H.F. is co-founder of Cambridge Display Technology and Heliochrome Ltd.

Acknowledgements

The authors thank the Engineering and Physical Sciences Research Council (EPSRC) and MBraun Inertgas-Systeme GmbH for support.

\section{Author Contributions}

R.H.F. leads the perovskite LED research programme at the Cavendish Laboratory (University of Cambridge). D.D. and R.H.F. planned and co-supervised the recent high-efficiency perovskite LED research. B.Z. and D.D. developed the high-efficiency LEDs. S.L. led the manuscript preparation and video production.

\section{Keywords}

Perovskite LED, best perovskite LED, OLED, commercial perovskite, perovskite review.

\section{Article Information}

This article is licensed by R. H. Friend et al. under a Creative Commons Attribution 4.0 International License (CC BY 4.0). 